\title{
Photodynamic Inactivation of Staphylococcus auerus by Novel Troponyl Methyl (Pyro)pheophorbides
}

\author{
Bayarmaa Bold, Bayarmaa Barkhuu, Woo-kyoung Lee, and Young Key Shim*
}

\begin{abstract}
PDT Research Institute, School of Nano Engineering. Inje Lniversity, Gimhae 612-749, Korea. " E-mail: viksimatinje ac kr Received Atrgust 24, 2007
\end{abstract}

Key Words : Antibacterial photodỵnamic inactivation. Troponỵl methỵl (pyro)pheophorbides

The emergence of antibiotic resistance among pathogenic bacteria has led to a major research effort to find the Photodynanic Therapy as an alternative antibacterial therapy

Bacterial photodynamic inactivation (PDI) is based on the adninistration of a photosensitizer. which is preferentially acciumulated in the microbial cells. The subsequent irradiation with visible light, in the presence of oxygen, specifically produces cell damages that inactivate the nicroorganisins.

PDI is known to be effective against viruses, bacteria, and fungi and therefore has been proposed to be used as a therapy for localized infections. ${ }^{1,3}$

It is known that Grant-positive bacteria are generally more susceptible to PDT as compared to Grant-negative species. This difference is explained by the structural differences in the cell walls. ${ }^{3,4}$

The novel candidates for PDI are based on methyl pheophorbide-a (MPa) and methyl pyropheophorbide-a (MPPa). isolated from alga Spintina pacifica. which were linked with tropolone. seven membered cyclic compound by substitution on 3-position.

Tropolone, as the substituent of new photosensitizers in this study, has been found to have the following biological activities: antimicrobial activity. cytotoxic effects on various human and nurine tumor cell lines. phytogrowth-inhibitory activity. insecticidal effect. metalloprotease inlibition, and antifungal activity on wood rotting fungi. $\$$

Meanwhile, chlorin-based compounds show effective anticancer photodynamic activities. ${ }^{7}$ Therefore, the combined conpound of novel photosensitizers as PDT and the tropolones as antinicrobials were prepared to see the dual function activities.

In this paper. we wish to report the preliminary results of the research on Grant-positive bacterial cell $S$. chirens (strain $\mathrm{KC}$ 1927) using troponyl methyl (pyro)pheophorbides.

Table 1. Newly synthesized photosensitizers

\begin{tabular}{lccl}
\hline Name & Entry & Chemical fonmula & MW \\
\hline $\mathrm{B}-1$ & $\mathrm{MPa}$ & $\mathrm{C}_{36} \mathrm{H}_{38} \mathrm{~N}_{4} \mathrm{O}_{5}$ & 606.7 \\
$\mathrm{~B}-11$ & 2 & $\mathrm{C}_{43} \mathrm{H}_{44} \mathrm{~N}_{4} \mathrm{O}_{5}$ & 728.85 \\
$\mathrm{~B}-12$ & 3 & $\mathrm{C}_{45} \mathrm{H}_{40} \mathrm{~N}_{4} \mathrm{O}_{3}$ & 770.94 \\
$\mathrm{~B}-2$ & $\mathrm{MPPa}$ & $\mathrm{C}_{34} \mathrm{H}_{3} \mathrm{~N}_{4} \mathrm{O}_{3}$ & 548.7 \\
$\mathrm{~B}-21$ & 5 & $\mathrm{C}_{41} \mathrm{H}_{4} \mathrm{~N}_{4} \mathrm{O}_{5}$ & 670.81 \\
$\mathrm{~B}-22$ & 6 & $\mathrm{C}_{44} \mathrm{H}_{48} \mathrm{~N}_{4} \mathrm{O}_{5}$ & 712.89 \\
\hline
\end{tabular}

Experiments were carried out in order to determine the antibacterial photodynamic activities of newly synthesized troponyl methyl (pyro)pheophorbides.

The data presented in Table 2 and Figures $1-2$ show that the illumination of $S$. correts cells which had been exposed to troponyl methyl (pyro)pheophorbide derivatives caused a considerable decrease cell survival, comparing to control conditions.

Our findings clearly demonstrate that newly synthesized troponyl methyl (pyro)pheophorbide derivatives (2,3 and 5, 6) exhibit significant antimicrobial photodynamic activities against Gram-positive bacterium $S$. aurets activity at micromolar concentrations.

Kill rate of bacterial cells increased in proportion to the increase of the incubation time and were depending on

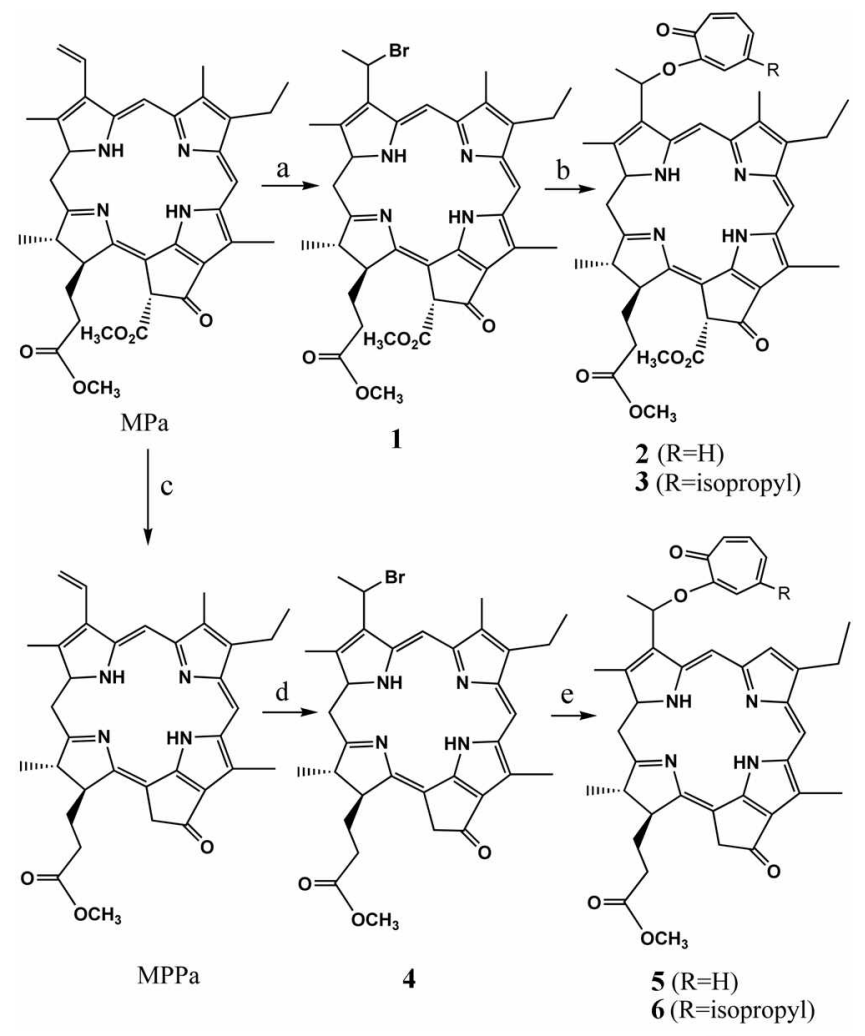

Scheme 1. Synthetic pathway of troponyl derivatives from MPPa and MPa. Reagents: a) $\mathrm{HBr} / \mathrm{AcOH}$, b) tropone $/ \mathrm{K}_{2} \mathrm{CO}_{3} / \mathrm{CH}_{2} \mathrm{Cl}$, c) collidine/reflux, d) $\mathrm{HBr} / \mathrm{AcOH}$, e) tropone $/ \mathrm{K}_{2} \mathrm{CO}_{3} / \mathrm{CH}_{2} \mathrm{Cl}_{2} \mathrm{R}=\mathrm{H}$, isopropyl 
Table 2. Cell killing results from the grouth delay experiments of $S$ aureus A. Methyl pheophorbide-a (MPa) and its derivatives

\begin{tabular}{|c|c|c|c|c|c|c|c|c|c|c|}
\hline \multirow{2}{*}{$\begin{array}{l}\text { Irradiance time } \\
\text { (min) }\end{array}$} & \multicolumn{2}{|c|}{$0.5 \mu \mathrm{M}$} & \multicolumn{2}{|c|}{$1, t, \mathrm{M}$} & \multicolumn{2}{|c|}{$2.5, \mathrm{tM}$} & \multicolumn{2}{|c|}{$5.0 / \mathrm{M}$} & \multicolumn{2}{|c|}{$10.0 \mu \mathrm{M}$} \\
\hline & CFU/112L & $\%$ & CFU/mLL & $\%$ & $\mathrm{CFU} / \mathrm{mL}$ & $\%$ & $\mathrm{CFU} / \mathrm{mL}$ & $\%$ & $\mathrm{CFU} / \mathrm{mL}$ & $\%$ \\
\hline \multicolumn{11}{|c|}{ Compound B- 1} \\
\hline 0 & $10.2 \times 10^{5}$ & 100 & $9.8 \times 10^{5}$ & 100 & $9.6 \times 10^{5}$ & 100 & $94 \times 10^{5}$ & 100 & $8.3 \times 10^{5}$ & 100 \\
\hline 15 & $9 \times 10^{5}$ & 88 & $8.1 \times 10^{5}$ & 83 & $7.6 \times 10^{5}$ & 79 & $6.4 \times 10^{5}$ & 68 & $4.5 \times 10^{5}$ & 54 \\
\hline 30 & $3.2 \times 10^{5}$ & 31 & $3 \times 10^{5}$ & 30 & $1.5 \times 10^{5}$ & 15 & $1.2 \times 10^{5}$ & 13 & $0.9 \times 10^{5}$ & 11 \\
\hline \multicolumn{11}{|c|}{ Compound B-11 } \\
\hline 0 & $5.5 \times 10^{5}$ & 100 & $4.1 \times 10^{5}$ & 100 & $3.6 \times 10^{5}$ & 100 & $2.3 \times 10^{5}$ & 100 & $1.3 \times 10^{5}$ & 100 \\
\hline 15 & $2.9 \times 10^{5}$ & 53 & $1.6 \times 105$ & 39 & $0.6 \times 10^{5}$ & 17 & $0.9 \times 10^{-1}$ & 3.9 & $0.2 \times 10^{4}$ & 1.6 \\
\hline 30 & $4.6 \times 10^{4}$ & 9 & $2.8 \times 10^{+}$ & 6.8 & $0.76 \times 10^{4}$ & 2.1 & $2.3 \times 10^{3}$ & 1 & $0.9 \times 10^{4}$ & 0.07 \\
\hline \multicolumn{11}{|c|}{ Compound B-12 } \\
\hline 0 & $1.9 \times 10^{5}$ & 100 & $1.4 \times 10^{5}$ & 100 & $1.1 \times 10^{5}$ & 100 & $0.8 \times 10^{5}$ & 100 & $0.37 \times 10^{5}$ & 100 \\
\hline 15 & $7.9 \times 10^{+}$ & 42 & $5.2 \times 10^{+}$ & 37 & $0.9 \times 10^{-1}$ & 8 & $0.2 \times 10^{3}$ & 2.5 & $0.1 \times 10^{3}$ & 0.27 \\
\hline 30 & $4.9 \times 10^{+}$ & 3 & $1 \times 10^{-2}$ & 0.71 & $0.69 \times 10^{3}$ & 0.6 & $0.72 \times 10^{7}$ & 0.09 & 0 & 0 \\
\hline
\end{tabular}

B. Methyl pyropheophorbide-a (MP'ra) and its derivatives

\begin{tabular}{|c|c|c|c|c|c|c|c|c|c|c|}
\hline \multirow{2}{*}{$\begin{array}{l}\text { Irradiance tinne } \\
\text { (min) }\end{array}$} & \multicolumn{2}{|c|}{$0.5 \mu \mathrm{M}$} & \multicolumn{2}{|c|}{$1 \mu \mathrm{M}$} & \multicolumn{2}{|c|}{$2.5 \mu \mathrm{M}$} & \multicolumn{2}{|c|}{$50 \mathrm{dM}$} & \multicolumn{2}{|c|}{$100, \mathrm{MM}$} \\
\hline & CFIJ/mI. & $\%$ & CFIJ/mI. & $\%$ & CFU/mL & $\%$ & CFIJ/TT, & $\%$ & $\mathrm{CF} J / \mathrm{mI}$ & $\%$ \\
\hline \multicolumn{11}{|c|}{ Compound B-2 } \\
\hline 0 & $9.5 \times 10^{5}$ & 100 & $9.1 \times 10^{5}$ & 100 & $8.1 \times 10^{5}$ & 100 & $6.5 \times 10^{5}$ & 100 & $6.1 \times 10^{5}$ & 100 \\
\hline 15 & $8.9 \times 10^{5}$ & 94 & $8.2 \times 10^{5}$ & 90 & $6.5 \times 10^{5}$ & 80 & $4.0 \times 10^{5}$ & 62 & $1.2 \times 10^{5}$ & 20 \\
\hline 30 & $6.9 \times 10^{5}$ & 73 & $3.6 \times 10^{5}$ & 40 & $2.7 \times 10^{5}$ & 33.3 & $1.1 \times 10^{5}$ & 17 & $0.3 \times 10^{5}$ & 5 \\
\hline \multicolumn{11}{|c|}{ Compound B-2 1} \\
\hline 0 & $8.5 \times 10^{5}$ & 100 & $7.6 \times 10^{5}$ & 100 & $4.5 \times 10^{5}$ & 100 & $2.1 \times 10^{5}$ & 100 & $1.4 \times 10^{5}$ & 100 \\
\hline 15 & $5.7 \times 10^{5}$ & 67 & $4.8 \times 10^{5}$ & 63 & $1.5 \times 104$ & 51 & $0.5 \times 10^{5}$ & 24 & $0.1 \times 10^{5}$ & 7.1 \\
\hline 30 & $3.5 \times 10^{5}$ & 41 & $1.7 \times 10^{5}$ & 22 & $0.02 \times 10^{5}$ & 9.5 & $0.02 \times 10^{5}$ & 1 & 0 & $u$ \\
\hline \multicolumn{11}{|c|}{ Compound B-22 } \\
\hline 0 & $8.0 \times 10^{5}$ & 100 & $7.8 \times 10^{5}$ & 100 & $6.0 \times 10^{5}$ & 100 & $6.5 \times 10^{5}$ & 100 & $0.3 \times 10^{5}$ & 100 \\
\hline 15 & $4.6 \times 10^{5}$ & 57.5 & $1.3 \times 10^{5}$ & 16.6 & $0.3 \times 10^{5}$ & 5.76 & $0.01 \times 10^{5}$ & 0.28 & $0.5 \times 10^{3}$ & 1.6 \\
\hline 30 & $1.3 \times 10^{5}$ & 16.3 & $0.3 \times 10^{5}$ & 3.8 & $0.01 \times 10^{5}$ & 0.19 & $0.26 \times 10^{3}$ & 0.07 & 0 & 0 \\
\hline
\end{tabular}
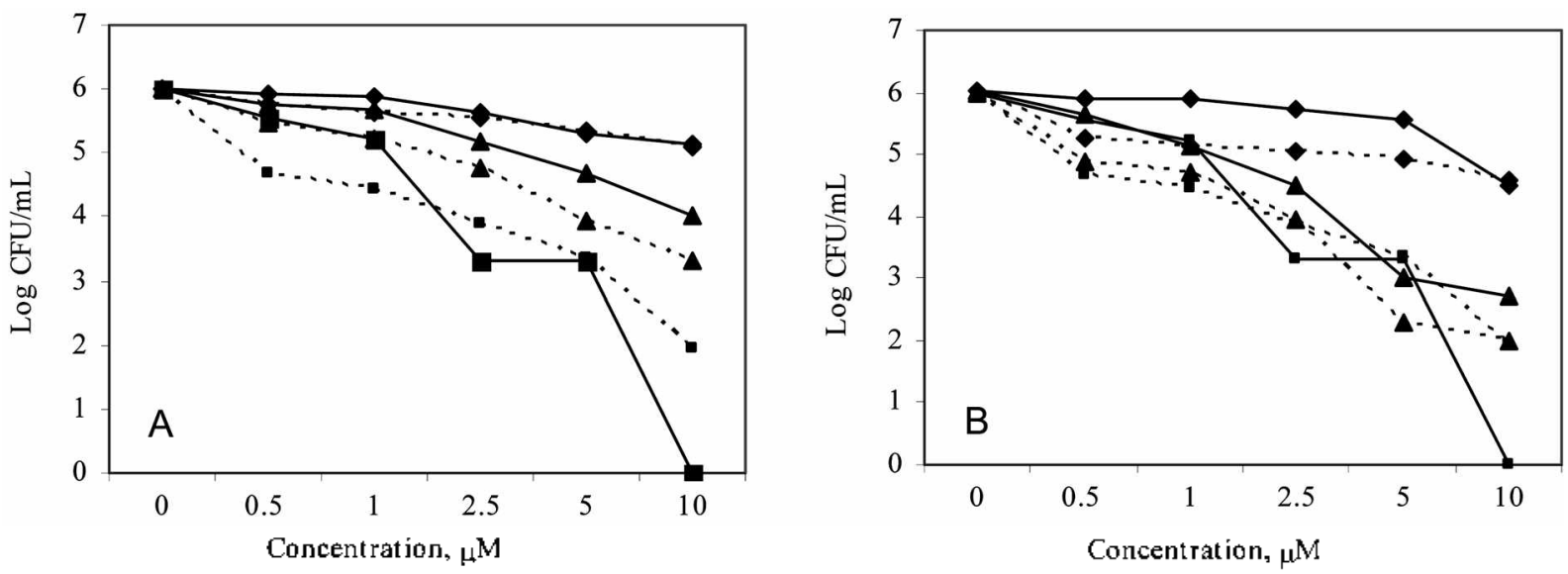

Figure 1. Survival curves of $S$. aurens incubated with various concentrations of newly synthesized B-1 series (A) and B-2 series (B) compounds and exposed to LED for 0, $15 \mathrm{~min}$, and $30 \mathrm{~min}$. A. Dotted line (Bll) and $\bullet$ - no light, $\mathbf{-}-15 \mathrm{~min}, \mathbf{-}-30 \mathrm{~min} ;$ black line (Bl2)

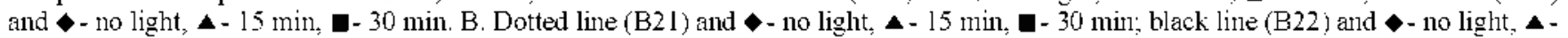
$15 \mathrm{~min}, \mathbf{0}-30 \mathrm{~min}$. Results are the mean values of at least three experiments.

chemical structure of the photosensitizing agents.

In the absence of irradiation, incubation with photosensitizers at concentrations of $5-10 \mu \mathrm{M}$ for $10 \mathrm{~min}$ at dark caused a slight decrease in number of CFU/mL. showing antibacterial activity of tropolone itself. As a result we see the dual function activities of photosensitizers linked with 

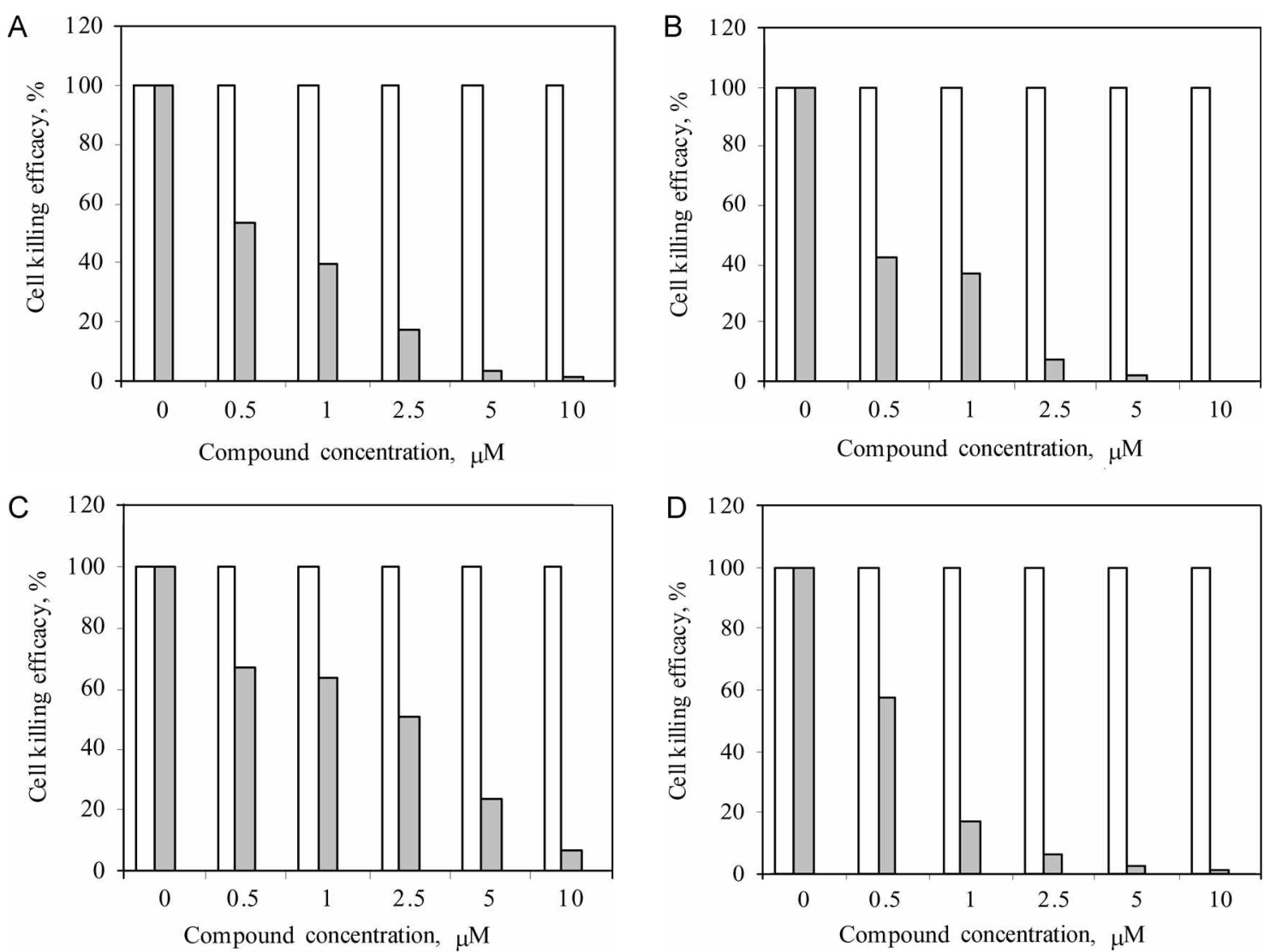

Figure 2. Cell killing efficacy of photosensitizers (A-B11, B-B12, C-B21, D-B22) against $S$, attrets after 10 min of incubation. Open bars, $S$ aureus cells after incubation with different concentrations without illumination; grey bars, $S$. ameus cells after incubation with different concentrations followed by illumination for $15 \mathrm{~min}$

tropolones over $5 \cdot 10 \mu \mathrm{M}$ concentration.

Pyropheophorbide derivatives are relatively better than pheophorbide derivatives and $30 \mathrm{~min}$ is enough to destroy bacterial cells completely less than $10.0 \mu \mathrm{M}$. Also isopropyl derivatives showed better inhibition than free tropolone. which may suggest more lipophilicity give better penetration for better inhibition.

The preliminary results presented indicate the promising activity of troponyl methyl (pyro)pheophorbide and its derivatives as potential candidates for antimicrobial photodýnamic inactivation.

At present. we are extending our antimicrobial photodynamic studies to other group of photosensitizers and the photoinactivation efficiency on various Gram-negative and Gram-positive bacteria.

\section{Experimental Section}

General procedure for the reaction of bromo-chlorin $\left(1,4^{s}\right)$ with tropolone. For each reaction, we prepared troponyl methyl pheophorbide-a $(2,3)$ and troponyl methyl py ropheophorbide-a. (5.6) The bromo-chlorin $(1.3 \mathrm{mmol})$ and tropolone $(1.5 \mathrm{mmol}$ ) were dissolved in dry dichloromethane $(2 \mathrm{~mL})$ with $\mathrm{K}_{3} \mathrm{CO}_{3}(3.0 \mathrm{~mm} / \mathrm{l})$ and stirred for $12 \mathrm{~h}$ in the dark. The reaction nixture was partitioned with dichloromethane and water. The obtained organic phase was washed with $1 \mathrm{~N} \mathrm{HCl}$ solution and brine, and dried over $\mathrm{Na}_{2} \mathrm{SO}_{4}$. The crude products were purified by short column chromatograply over silica gel using $5 \%$ methanol in dichloromethane as the eluent to give the title conpounds 30 $50 \%$ yield

Selected data, for 2: UV-vis $\left(\mathrm{CH}_{2} \mathrm{Cl}_{2}\right) \lambda_{\text {max }}=664$ (relative intensity, 0.70), $606(0.12) .536(0.15), 504(1.15) .410$ (1.81) $\mathrm{nm}:{ }^{1} \mathrm{H}$ NMR (300 MHz. $\mathrm{CDCl}_{3}$ ): $\delta 9.52,9.38,8.55$ (each $1 \mathrm{H}$. s. meso-H). $7.58-7.43(5 \mathrm{H}, \mathrm{m}$. tropolone-H), 6.75 . $6.24(1 \mathrm{H} . \mathrm{s} .132-\mathrm{H}) .4 .44 .4 .19(2 \mathrm{H} . \mathrm{m} .17 .18-\mathrm{H}) .3 .85$. 3.64. 3.57. 3.53. 3.37 (each $3 \mathrm{H}$. s. Me+OMe). $3.64(2 \mathrm{H} . \mathrm{m}$. $81-\mathrm{H}) .2 .80,2.59(4 \mathrm{H}, \mathrm{m}, 171,172-\mathrm{H}), 2.24(3 \mathrm{H}, \mathrm{d}, J=6.4$ Hz. 32-Me). $1.79(3 \mathrm{H} . \mathrm{d} . J=7.2 \mathrm{~Hz} .18-\mathrm{Me}) .1 .67$ (3H. t. $J=$ $7.6 \mathrm{~Hz} .82-\mathrm{Me}) .0 .48-1.63(2 \mathrm{H}$. br s. $\mathrm{NH}$ ).

For 3: UV-vis $\left(\mathrm{CH}_{2} \mathrm{Cl}_{2}\right) \lambda_{\text {max }}=663.7$ (relative intensity. $0.62), 606(0.09) .536(0.11) .506(0.14), 410(1.62):{ }^{1} \mathrm{H}$ NMR $\left(300 \mathrm{MHz}, \mathrm{CDCl}_{3}\right), \delta: 9.51,9.38 .8 .56$ (each, IH. s, meso-H), 7.84-7.62 (4H. m, $\beta$-thujaplicin-H). 6.85. 6.27 (IH. s, 132-H), 4.46, 4.19 (2H, m, 17. 18- H), 3.84, 3.66, 3.54.3.37 (each 3H. s. Me+OMe). $3.81 .3 .64(2 \mathrm{H} . \mathrm{m} .81-\mathrm{H})$. $2.85 .2 .62(4 \mathrm{H}, \mathrm{m} .171 .172-\mathrm{H}), 2.17$. (3H. d. $J=6.4 \mathrm{~Hz} 32-$ Me). $1.79(3 \mathrm{H} . \mathrm{d} . J=7.2 \mathrm{~Hz}, 18-\mathrm{Me}) .1 .68(3 \mathrm{H}$. t. $J=7.6 \mathrm{~Hz}$. $82-\mathrm{Me}$ ). 1.34 (d. $6 \mathrm{H} . J=6.8 \mathrm{~Hz}$ ). $0.32-1.49(2 \mathrm{H}$. br s. NH).

Selected data, for $5: \mathrm{UV}$-vis $\left(\mathrm{CH}_{2} \mathrm{Cl}_{2}\right) \lambda_{\text {max }}=663.4$ (relative intensity, 0.79), 606 (0.14). $535(0.17), 509(0.18) .409$ (2.04) um: ${ }^{1} \mathrm{H}$ NMR $\left(300 \mathrm{MHz}, \mathrm{CDCl}_{3}\right) ; \delta 9.52,9.38,8.55$ 
(each $\mathrm{IH}$. s, meso-H), 7.58-7.41 (5H. m, tropolone-H), 6.75 . $6.24(\mathrm{lH} . \mathrm{s}, 132-\mathrm{H}), 4.44 .4 .19(2 \mathrm{H} . \mathrm{m}, 17,18-\mathrm{H}), 3.64$. $3.57,3.53,3.37$ (each $3 \mathrm{H}, \mathrm{s}, \mathrm{Me}+\mathrm{OMe}) .3 .64(2 \mathrm{H} . \mathrm{m}, 8 \mathrm{l}-\mathrm{H}$ ). $2.80,2.59(4 \mathrm{H} . \mathrm{m}, 17 \mathrm{l}, 172-\mathrm{H}), 2.24$, (3H. d. $J=6.4 \mathrm{~Hz}, 32-$ $\mathrm{Me}), 1.67(3 \mathrm{H}, \mathrm{d} . J=7.2 \mathrm{~Hz}, 18-\mathrm{Me}), 0.48-1.63(2 \mathrm{H}$, br s, NH).

For 6: UV-vis $\left(\mathrm{CH}_{2} \mathrm{Cl}_{2}\right) \lambda_{\text {max }}$ : 664.8 (relative intensity. $0.66), 606(0.78), 535(0.77), 505(0.86), 410(0.94) \mathrm{lm} ;{ }^{1} \mathrm{H}$ NMR $\left(300 \mathrm{MHz}, \mathrm{CDCl}_{3}\right) \delta 9.60 .9 .40 .8 .54$ (each IH. s. meso-H). 7.99-7.89 (4H. m, $\beta$-thujaplicin-H), 6.75. 6.24 (1H, s. 132-H). 4.44. 4.27 (2H. m. 17, 18-H). 3.64. 3.59. $3.43,3.37$ (each $3 \mathrm{H}, \mathrm{s}, \mathrm{Me}+\mathrm{OMe}) .3 .84(2 \mathrm{H}, \mathrm{ml} .81 \mathrm{H}) .2 .90$. 2.65 (4H. m, 171, 172H). 2.25 (3H. d, $J=6.4 \mathrm{~Hz}, 32-\mathrm{Me})$ $1.78(3 \mathrm{H}, \mathrm{d} . J=7.2 \mathrm{~Hz} .18-\mathrm{Me}), 1.68(3 \mathrm{H} . \mathrm{t}, J=7.6 \mathrm{~Hz} .82-$ Me). 1.31 (d. $6 \mathrm{H}, J=6.8 \mathrm{~Hz}) .0 .48-1.70(2 \mathrm{H}$. br. $\mathrm{NH})$.

Antimicrobial activity. The cytotoxic action of troponyl methyl (pyro)pheophorbide derivatives on bacterial cells was assessed after 10 min of incubation in the dark. The irradiance was performed by a LED light source UFPh-630/ 675-01-BIOSPEC (BioSpec. Russia) for 15 and $30 \mathrm{~min}$ on the plate containing the microbial cell suspension at the top of $20 \mathrm{cml}$.

Survival fractions for the PDI experinents were expressed as the ratios of CFU from cultures treated with both light and compound to CFU measured for cultures treated with a compound or a light. ${ }^{\text {li... }}$
Acknowledgment. This work was supported by research grant from PDT Research Institute, Inje University.

\section{References}

1. Hamblin. M. R.: Hasan1. T. Photochem. Photobiol. Sci. 2004. 3. $436-450$.

2. Jor, G: Brown. S. Photochem. Photobiol. Sci 2004. 3. 403405 .

3. Caminos. D. A.: Spesia. M. B.: Durantini. E. N. Photochent Photobiol. Sci. 2006. 5. 56-65.

4. Jori. G.: Fabris. C.: Soncin. M.: Ferro. S.: Coppellotti. O.: Dei. D.: Fantetti, L: Chiti, G.; Roneueci. G. Lasers in Surgeny and Medicine 2006. 38. $468-481$

5. Morita Y.: Matsumura, E.: Okabe. T. Biol. Phom. Bul. 2003. 26. 1487-1490.

6. Trust. T. T. Antinicrob. Agents and Chentother 1975. 7. 500 506.

7. Webber, J.: Leeson, B.; Fromm, D.: Kessel. D. I. Photochem. Photobiol 2005, 78. 135-140.

8. Wang, J.-J; Han. G.-F.: Wu. X.-R.: Wang, L.-M; Shim. Y. K Chin. J. Org Chem. 2004. 2+(5). 537-542.

9. Demidova. T. N.: Hamblin. M. R. Antinticrob. Agents and Chenother. 2005. 49(6). 2329-2335.

10. Minnock. A.: Vernon. D. A.: Schofield. J.: Griffiths, J.; Parish, J. $\mathrm{H} ;$ Brown. J. S. Photochem. Photobiol. B 1996. 32. 159-164.

11. Hamblin. M. R: O'Donnel. D. A.: Murthy. N.; Rajagopalan. K: Michaud. N.: Sherwood, M. E.: Hasan, T. J. Antimicrob. Chentother 2002. $49.941-951$ 